\title{
SECURITY ANALYSIS OF MOBILE AUTHENTICATION USING QR-CODES
}

\author{
Siwon Sung ${ }^{1,2}$, Joonghwan Lee ${ }^{1,2}$, Jinmok Kim ${ }^{1,2}$, Jongho Mun ${ }^{2}$ and \\ Dongho Won ${ }^{2}$ \\ ${ }^{1}$ Samsung Electronics, Suwon, Korea \\ \{siwon.sung, joonghwan.lee, jinmok.kim\}@samsung.com \\ ${ }^{2}$ College of Information and Communication Engineering, \\ Sungkyunkwan University, Suwon, Korea \\ \{jhmoon, dhwon\} esecurity.re.kr
}

\begin{abstract}
The QR-Code authentication system using mobile application is easily implemented in a mobile device with high recognition rate without short distance wireless communication support such as NFC. This system has been widely used for physical authentication system does not require a strong level of security. The system also can be implemented at a low cost. However, the system has a vulnerability of tampering or counterfeiting, because of the nature of the mobile application that should be installed on the user's smart device. In this paper we analyze the vulnerabilities about each type of architectures of the system and discuss the concerns about the implementation aspect to reduce these vulnerabilities.
\end{abstract}

\section{KEYWORDS}

Authentication, Security, QR-Code, Mobile

\section{INTRODUCTION}

The one-dimensional barcodes have been used in limited areas such as identifier of goods due to the limitation of information capacity. The two-dimensional barcode has been widely used in various fields because of great increase of the information capacity. Thus the user authentication system using the QR-Code becomes possible. Therefore the widely use of smart devices has promoted to spread the QR-Code authentication system. Users could generate the QR-Code image for authentication easily through mobile application on their smart devices. The system is mainly used in a low cost authentication system such as gate access control in buildings and unmanned book rental system in libraries. However, this system is vulnerable to attempts to disguise access to someone else's identity because of the nature of easily replicable QR-Code. In case of mobile device, it is very easy to duplicate through screen capture and be transferred to other user via instant messenger. Thus it is very difficult to eradicate these user violations because of the limitations by those characteristics. In order to overcome these vulnerabilities, various considerations such as security policy, authentication protocols, network security are required. In addition, the system needs also to consider reverse engineering because the vulnerable attributes of the mobile application. The reverse engineering can occur on the various components to configure the system. Particularly the mobile application running on the smart

David C. Wyld et al. (Eds) : NETCOM, NCS, WiMoNe, CSEIT, SPM - 2015

pp. 151-160, 2015. @ CS \& IT-CSCP 2015

DOI : $10.5121 / \mathrm{csit} .2015 .51612$ 
device platform is extremely vulnerable to reverse engineering. Even if an application use very secure authentication scheme, the security might not meet the security levels by lower implementation maturity. Whereas the system uses vulnerable methods, fine implementation recommendations help to prevent those vulnerabilities. In this paper, we suggest some recommendations in implementation points of view to prevent those threats. The remainder of the paper is organized as follows. In Section 2, related works are presented. We introduce the general architecture of the QR-Code authentication system using mobile application in Section 3. The attacker models are described in Section 4. The vulnerabilities in the software implementation point of the view are discussed in Section 5. The result of the forgery attack on real system is reported in Section 6. Then the countermeasure to reduce this software threats is given in Section 7. Finally, we conclude this paper in Section 8.

\section{RELATED WORK}

QR-TANs is a transaction authentication technique based on QR-Code, allow the user to directly validate the content of a transaction within a trusted device [1]. Liao et al. [2] proposed a scheme based on two-dimensional barcode not only eliminates the usage of the password verification table, but also is a cost effective solution. Lee et al. [3] proposed an authentication system used Mobile OTP with the combination of QR-Code. Oh et al. [4] suggests a technology to authorize users three kinds of QR-Code. Kao et al. [5] try to develop a safe and efficient authentication way by using mobile device and implement it for access control system that enhances the security of physical access control systems. 2CAuth is a two factor authentication scheme that enhances secure usage of application information and preserves usability, without sacrificing user's privacy [6]. Lee et al. designed secured $Q R$-Login user verification protocol for smart devices that are ready to communicate with QR-Code and proposed a way to keep critical data safe when using the Internet [7]. Kale et al. proposed a anti phishing single sign-on authentication model using QR-Code [8]. Malik et al. introduced the idea of a one-time password, which makes unauthorized access difficult for unauthorized users [9].

\section{ARCHITECTURE OF AUTHENTICATION SYSTEM}

The QR-Code authentication system using mobile application (BAS-MA) consists of five participants: the users $\mathrm{U}$, the mobile application $M A$, QR-Code generator $Q G$, the service provider $S P$ and authentication center $A C$. We summarize the notations and acronyms used in this paper in Table 1.

Table 1. Notations and Acronyms

\begin{tabular}{cl}
\hline$U_{i}$ & User \\
$C_{i}$ & User credentials for $U_{i}$ \\
$B_{i}$ & QR-Code for authentication issued to $U_{i}$ \\
$Q G$ & QR-Code generator \\
$S P$ & Service provider \\
$A C$ & Authentication center \\
& \\
$B A S-M A$ & QR-Code authentication system using mobile application \\
$A C-A R$ & Authentication center based architecture \\
\hline
\end{tabular}




\begin{tabular}{cl}
\hline$M A-A R$ & Mobile application center based architecture \\
$A_{i t}$ & Internal attacker \\
$A_{e x}$ & External attacker \\
\hline
\end{tabular}

$B A S-M A$ is classified into two kinds of architectures based on the location of the $Q G$ :

- Authentication Center based Architecture

- Mobile Application based Architecture

In the $A C-A R, Q G$ is located in $A C$ that could be regarded as a secure server in the remote location. The basic configuration of $A C-A R$ is shown in Figure 1. The procedure through which a $U_{i}$ can get a valid access to $S P$ is the following:

1. $U_{i}$ is willing to use the $S P . U_{i}$ get authentication through the user authentication scheme of the system.

2. $M A$ requires $B_{i}$ for authentication to $A C$.

3. $A C$ retrieves user data from User Data Storage then calculates $C_{i}$. $A C$ asks the $Q G$ a $B_{i}$ carrying $C_{i}$.

4. $Q G$ transform the $C_{i}$ to $B_{i}$ without any modification on $C_{i}$ and returns a $B_{i}$.

5. $A C$ passes through the $B_{i}$ to $M A$ via secure channel.

6. $M A$ displays the $B_{i}$ on its screen and submits to $S P$.

7. $S P$ decodes the $B_{i}$ and extracts $C_{i}$. $S P$ asks the $A C$ the verification carrying the $C_{i}$.

8. $A C$ verifies $C_{i}$ and return with an authentication callback.

The $B_{i}$ is generated in the $A C$ on remote. The $M A$ has just downloaded the image $B_{i}$ from $A C$ and displays $B_{i}$ on its screen. The $M A$ does not have any logic for generating $B_{i}$ or processing $C_{i}$ and is just provided with a bitmap image. If the $M A$ is authorized to download the $B_{i}$, the $C_{i}$ can be secured to the reversing attack because they are created and managed on $A C$ in remote.

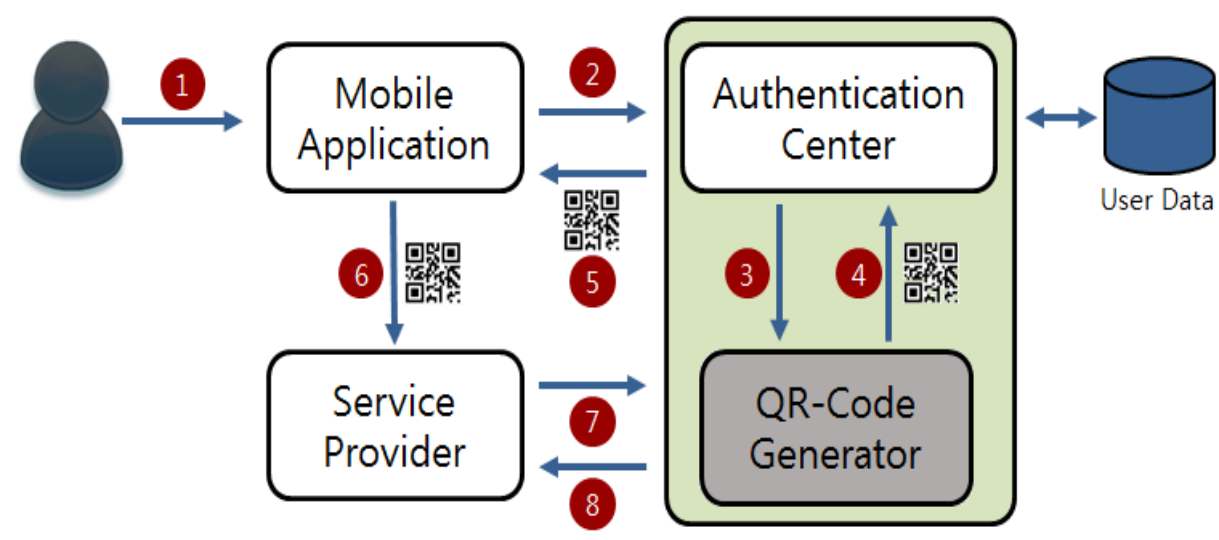

Figure 1. Basic procedure of Authentication Center based Architecture

Otherwise, the $Q G$ is associated with $M A$ tightly in $M A-A R$. The $M A$ includes $Q G$ itself or is able to interact with the external $Q G$ in the same device. Figure 2 shows the operation flows that the 
$M A$ is granted the permission for $S P$. The procedure on $M A-A R$ is different to that of the $A C-A R$, yet it has the following differences:

1. $U_{i}$ try to get a permission to access $S P$.

2. $M A$ requests a $C_{i}$ to generate $B_{i}$.

3. AC assemble $C_{i}$ using the User Data Storage and return to $M A$.

4. $M A$ askes $Q G$ existed in mobile device a $B_{i}$ carrying $C_{i}$.

5. $Q G$ generate $B_{i}$ containing $C_{i}$ then pass it to $M A$.

6. $M A$ gives the $B_{i}$ to $S P$.

7. $S P$ open the $B_{i}$ and find $C_{i}$ then $S P$ request a verification to the $A C$ with the $C_{i}$.

8. $A C$ checks the equality of the $C_{i}$ and response.

The $M A$ is responsible to manage the $C_{i}$ in secure and to generate the $B_{i}$ itself. The $Q G$ is exposed to threat to get to be attempt software attack.

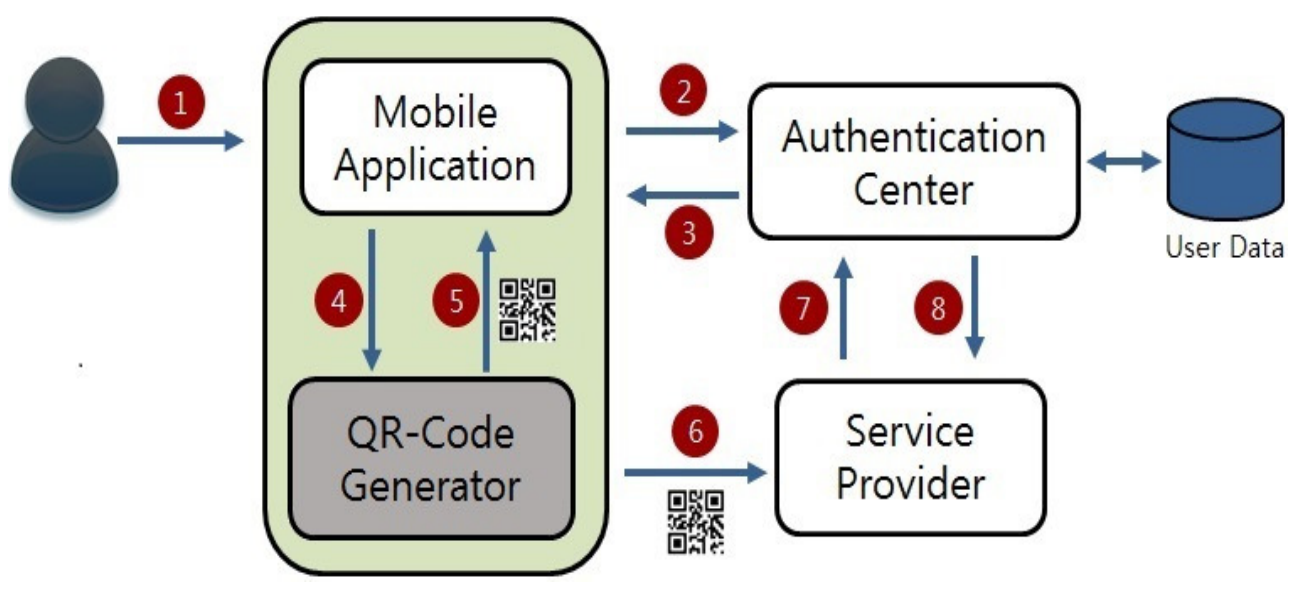

Figure 2. Basic procedure of Mobile Application based Architecture

\section{ATTACK Motels}

The attackers who threaten the $B A S-M A$ can be divided into two groups:

\subsection{The Internal Attacker}

The internal attacker $A_{i t}$ is a member of the organization provided services by $S P$ and has the correct permissions can access the $S P$. $A_{i t}$ may be a malicious user himself willing to access to $S P$ by the identity of the other user $U_{j}$. The $A_{i t}$ also tries to let an unauthorized person to get the authentication to the system by sharing his identity. The $A_{i t}$ can try various attacks on the system because they know very well about the $B A S-M A$, and can examine the $M A$ on their mobile devices. $A_{i t}$ is able to perform repeated incorrect attempts to authentication system with less doubt of the system administrator. 


\subsection{The External Attacker}

Otherwise, the external attacker $A_{e x}$ is not participated in the organization. $A_{e x}$ is the attacker would like to acquire authentication to the system even if they don't have any right permissions. Depending on the mobile application distribution policy, the $A_{e x}$ may have difficulties to obtain the installable packages or binary of the mobile applications. For that reason, $A_{e x}$ distribute a malware to find vulnerabilities in order to steal someone's $C_{i}$ and $B_{i}$.

\section{VULNERABILITY ANALYSIS}

\subsection{QR-Code Cloning}

In the $B A S-M A$, the $B_{i}$ is the key object to achieve the authentication. However, the $B_{i}$ could be cloned very easily on $M A$ through screen capture. The $A_{i t}$ might cause the masquerade attack transferring their image to others via an instant messenger. The $A_{e x}$ also try to get the copies of the $B_{i}$. In fact, it is impossible to completely prevent such cloning because of the nature of the QR-Code that should be exposed on the screen. The $A_{e x}$ could even take a picture of the $B_{i}$ using a separate camera.

\subsection{Authentication Hijacking}

The permanent authentication such as automatic login is usually enabled for the convenience use after once successful authentication. Even if the authentication protocol do not support long term session, the developer tries to implement virtual session by background authentication. In order to do it, the keeping of the user authentication cookies such as user's identity and password in the internal cache are required. These important data in the cache can be easily exposed by the software attack. The attackers are able to hijack the user authentication through the cached data.

\subsection{Stored Data Exfiltration}

The $C_{i}$ is stored in the non-volatile storage area under $M A$ in the $M A-A R$. The attackers can extract the values through software attack. If the device is rooted, the attack is easier to occur. Because of that, it is recommended that the value is not stored permanently or data should be stored encrypted. Once the encryption key is exposed, the protection using cryptography is useless. After that, the attackers are able to generate the $B_{i}$ without big difficulty. The data in local storage is very vulnerable. In rooted device, the malware get the read permission under other application's control. The attacker also can access the file system on the device directly or inspect the runtime memory.

\subsection{Internal Algorithm Disclosure}

The developers usually assume that the compiled source code will be safe from the logic disclosure. However, the powerful tools such as decompiler and logic analyzer are able to disclose the internal logic. The decompiler is able to recover the source codes using the binaries. The logic analyzer can draw the flow graph to help the understanding the logic. It can expose which cryptographic algorithms are used and how to assemble the parameters to construct credentials. In case of the use of the original designed two-dimensional barcode formats for the security purpose, the algorithms should not be disclosed and the software modules for the algorithms should not to be exposed and extracted for other application. If it is possible, the attackers can produce forgery algorithms to perform same work. 


\subsection{Network Message Eavesdropping}

The attackers have various methods to intercept the messages on the mobile device. The general purpose smart devices can be installed any application without any restrictions. The $A_{i t}$ could install a network packet monitoring tool on their mobile device to eavesdrop the messages between $M A$ and the $A C$. While the mobile platform operates under some policies that restrict the packet monitoring permissions, the $A_{i t}$ can attempt to bypass the restriction by using rootkit. In rooted device, all application could obtain root permission.

\subsection{IPC Message Eavesdropping}

In the $M A-A R$, the $Q G$ can be located in separated module from $M A$. This is the case in order to use the common module to generate barcode provided by the platform. In this case, the IPC (Inter Process Communication) communication is generally used. If the IPC channel is not secure, messages $C_{i}$ through $I P C$ can be exposed to malware. For example, the Broadcast is popularly used in Android platform to pass the values to other application. However the implicit broadcast message could be broadcasted to all the application in the device not excluding malwares.

\subsection{Communication Protocol Vulnerabilities}

The secure protocols such as $S S L / T L S$ prevent the leakage of the network packet. In this case, the attacker can manipulate the victim's network packet to pass through the malicious HTTP Proxy to incapacitate the secure communication. The HTTP Proxy can perform the man-in-the-middle attack to obtain the secrete key of the channel. Callegati et al. presented the man-in-the middle attack to the HTTPS through ARP Spoofing [10]. $A_{e x}$ also can install rogue access point in the near place that the authentication communication usually happened. Because the authentication procedure has occurred in fixed places such as the gateway of the buildings or in front of the unmanned machines, $A_{e x}$ could intercept the communications to proceed authentication through the rogue access point. Marlinspike et al. showed SSL Strip Attack that do a man-in-the-middle attack on SSL connections [11].

The Internal logic for communication in the software should be hidden as much as possible. The address of the destination server, Restful API names, the configuration of the parameters and the keyword for the message format are usually hard-coded inside mobile applications. The skilful attacker can reconstruct the protocol completely using a piece of this information.

\section{FORGERY ATTACK RESULTS}

We performed a forgery attack based on the discussed vulnerability in the previous section against the real world $B A S-M A$. The used attacker model was the internal attacker $A_{i t}$ and the architecture of the targeted system was $M A-A R$. The $A_{i t}$ registered his device to the $A C$ through user authentication phase. Then the $M A$ downloaded the $C_{i}$ and generated $B_{i}$ for displaying. The $B_{i}$ had expired in 5 minutes to prevent replay attack. The $M A$ was able to create a new $B_{i}$ every 5 minutes without additional network communication with $A C$. This means that the targeted $M A$ had stored not only user's credential but also the refresh logic in itself. The targeted $B A S-M A$ also used its original two-dimensional barcode format (Inter-Code). The attack procedure is described as follows. The $A_{i t}$ intercepted the packets of the user authentication phase. The packets were not encrypted and the network channel also wasn't secure. $A_{i t}$ could find a plain XML document containing $C_{i}$ in the intercepted packets. The $C_{i}$ had the member identification number and 
organization code. The $A C$ received the user's mobile phone number as parameter then returned the $C_{i}$. Anyone with a member's phone number can obtain member's credential data. The $Q G$ was included in the install package of the $M A$. The binary package of $M A$ could be extracted from the targeted device without root permissions. The $A_{i t}$ could obtain the $Q G$ by uncompressing the package. The $Q G$ was a kind of shared object for the targeted device platform. $A_{i t}$ tried to decompile the binaries to understand the interface and the parameters of the $Q G$. Thus the interface module was implemented in Java, the analysis didn't need high difficulty. The free decompile and analysis tools were available without commercial license. $A_{i t}$ could find the logic that how to assemble the $U_{i}$ and timestamps to make the parameters for the $Q G$. The shared object could be linked and executed with the forgery application on the same platform. Finally, $A_{i t}$ could produce the forgery application that received the victim's mobile number as parameter, then displayed the cloned $B_{i}$ as Inter-Code format. The $B_{i}$ could be accepted by the verification on the real world $B A S-M A$ without any restrictions.

\section{RECOMMENDATIONS FOR SECURE IMPLEMENTATION}

In Section 5, the various software vulnerabilities on the BAS-MA caused by immaturity implemented were discussed. The case study of the forgery attack through the software vulnerabilities on real system was reported in Section 6. Based on this, we suggest the countermeasures against the premature software implementations.

\subsection{Interfered Screen Capturing}

The screen capture function is usually enabled on common smart devices. It is recommended to block the function while the $M A$ shows the $B_{i}$. The watermarking on the captured image is also good to give a warning to the $A_{i t}$. It might be able to stop the harmful behaviour of the $A_{i t}$.

\subsection{Expiring QR-Code Available Period}

The expiration of already issued $B_{i}$ could cancel the replay attack. Some of salt value has to be mixed with the $C_{i}$ before $Q G$ generates the $B_{i}$. Lee et al. [7] proposed a timestamps based authentication scheme suited for mobile device environment, in which users can be authenticated using a QR-Code. A secure authentication system proposed by Shamal et al. [12] that uses a two factor authentication by combining a password and a camera equipped mobile phone, where mobile phone is acting as a authentication token. If the nonce for expiring is hard-coded in the $M A$, it should be obfuscated against to be disclosed by malicious inspection.

\subsection{Maintain Local Storage Cleanliness}

The candidate data willing to be stored in local storage should be reviewed carefully. The local storage is always in danger to be examined by attackers. If it is possible, the empty of the local area is recommended. The session between $M A$ and $A C$ should be implemented based on secure protocol. The developer should not arbitrarily implement features beyond the protocol.

\subsection{Deliberate Data Storing}

The cryptography does not guarantee complete data protection. However, storing data without encryption is more dangerous. The encryption key supplied from trusted party over secure channel is better than included in the binary package. The white-box cryptography helps to hide the key inside the application [13]. If the decryption of the stored value is not required, one-way 
hash function could be a good choice. In other hand, hardware-backed approach such as ARM TrustZone [14] offers a high level security assurance. It provides a completely isolated memory area and data storages physically or logically. However, the application requires a close collaboration with hardware manufacturers to use this approach, and there are many restrictions on the implementation.

\subsection{Obfuscation}

The software obfuscation is a process to transform the source code into obfuscated code. The variable names are replaced by meaningless name and the execution flows become skewed without any logical error. It is a time consuming and laborious process to analyze the obfuscated binary. The obfuscation is the most effective and comprehensive defence against reversing attack. The obfuscation tool produces a obfuscated result with the source code or compiled binary. This can be fully integrated and automated build process. In fact, the developers do not need to worry to apply the obfuscation. It can be easily accomplished using the 3rd-party tools. In Android, the ProGuard is included as default in build system. Ensure good performance, it is good to use a commercial tool. The high performance obfuscation tools replace the strings, the constants and the hard-coded encryption keys by encrypted themselves. The obfuscation of control-flow had been proposed by Chow et al. [15]. In case that the $M A$ includes the encryption key, the obfuscated use of key storage is the essential of the successful encryption.

\subsection{Securing Messaging over IPC}

When the mobile application structure uses the external $Q G$ through IPC, the IPC message carrying $C_{i}$ to $Q G$ has to be attention not to be eavesdropped. According to the type platforms, the secure communication between processes might not be supported. The $C_{i}$ should not be sent through a non-secure IPC. Chin et al. examine Android application interaction and identify security risks in application components. They provide a tool, ComDroid, that detects application communication vulnerabilities [16]. The TaintDroid is an extension to the Android mobile-phone platform that tracks the flow of privacy-sensitive data through third-party applications [17].

\subsection{Detecting Rootkit and Malware}

The attack on the rooted device is always more critical. The restricted policies are able to be cancelled easily. The applications are free to invade the protected area each other. It is recommended that let $M A$ not launch on the rooted device. A rootkit is a set of malicious tools, in order to achieve the top level privilege of the system. Kruegel et al. presents a technique that exploits binary analysis to ascertain, at load time [18]. In case of the difficulty to implement to detect rootkit or malware itself, the use of 3rd-party solution could be alternative.

\subsection{Detecting Rogue Access Point}

The rogue AP is a wireless access point installed on a wired enterprise network without authorization from the network administrator. They are installed by a legitimate user who is unaware of its security implications or easily smuggled onto enterprise premises by an outsider. It also allows the attacker to conduct a man-in-the-middle attack. The white-list based detection approach [19] is appropriate to BAS-MA. The valid access point nearby $S P$ could be listed up. It also can be an alternative to use the mobile network only such as $3 G$ or LTE instead of WIFI. 


\subsection{Restricting Allowed Device}

The number of authentication enabled devices should be limited against the hijacking. Thus the $B_{i}$ could be downloaded in only predefined mobile device. The device unique key is useful to implement the restrictions. The IMEI (International Mobile Station Equipment Identity) is a unique number to identity $3 G P P$ (GSM, LTE) mobile device. The IMEI could be a seed of the device unique key. The key is transmitted to the server along with the request for $B_{i}$. The server verifies the equality of the unique key from mobile device and the registered key in the server. Therefore, even if an attacker acquires a login cookie, it cannot be used in other devices. Of course the device unique key should not be stored in the local cookies.

\subsection{Forged Verification}

The executable is recommended to be signed by trusted certificate authority. This allows that the platform is able to verify whether the binary is forged. The application can verify the certification itself using platform API. The signature of the binary package could be accessed using the PackageManager in Android.

\subsection{Limiting Distribution Path}

The public on-line application store such as Google Play Store can be accessed without any limitation to download some mobile applications registered in the store. If the $M A$ is registered on the public store, $A_{e x}$ can download and install on their own devices in order to analyze the internal structures. The applications for internal users, such as $M A$ are recommended to distribute on a private path. The system operators can send a URL containing the application downloadable link to the valid user via $S M S$ or E-mail.

\section{CONCLUSION}

We discussed the vulnerabilities on the QR-Code authentication system caused by an immature software implementation. The main issue of the software security on the QR-Code authentication system is the protection of the logic and data around the QR-Code generator. These vulnerabilities can be overcome by a mature implementation. The improvisation implementation beyond the specification of the protocol is to be eradicated. The locally stored data should be cherry-picked and encrypted. The obfuscation can complete the robust software implementation. With these recommendations, the QR-Code authentication system would be a good solution for physical access system.

\section{REFERENCES}

[1] Starnberger, G., Froihofer, L., \& Göschka, K. M., (2009) "QR-TAN: Secure mobile transaction authentication", Availability, Reliability and Security, pp578-583.

[2] Liao, K. C., \& Lee, W. H., (2010). "A novel user authentication scheme based on QR-code" Journal of Networks, No.5(8), pp937-941.

[3] Lee, Y. S., Kim, N. H., Lim, H., Jo, H., \& Lee, H. J., (2010) "Online banking authentication system using mobile-OTP with QR-code”, Computer Sciences and Convergence Information Technology, pp644-648.

[4] Oh, D. S., Kim, B. H., \& Lee, J. K., (2011) “A study on authentication system using QR code for mobile cloud computing environment", Future Information Technology, pp500-507

[5] Kao, Y. W., Luo, G. H., Lin, H. T., Huang, Y. K., \& Yuan, S. M., (2011) "Physical access control based on QR code" Cyber-enabled distributed computing and knowledge discovery pp285-288 
[6] Harini, N., \& Padmanabhan, T. R., (2013) "2CAuth: A new two factor authentication scheme using QR-code”, International Journal of Engineering and Technology, No.5, pp1087-1094.

[7] Lee, Y., Kim, J., Jeon, W., \& Won, D., (2012) "Design of a simple user authentication scheme using QR-code for mobile device", Information Technology Convergence, Secure and Trust Computing, and Data Management, pp241-247.

[8] Kale, V., Nakat, Y., Bhosale, S., Bandal, A., \& Patole, R. G., (2015) "A Mobile Based Authentication Scheme Using QR Code for Bank Security"

[9] Malik, J., Girdhar, D., Dahiya, R., \& Sainarayanan, G., (2014) "Multifactor Authentication Using a QR Code and a One-Time Password", Journal of Information Processing Systems, No.10.

[10] Callegati, F., Cerroni, W., \& Ramilli, M., (2009) "Man-in-the-Middle Attack to the HTTPS Protocol", IEEE Security \& Privacy, pp78-81.

[11] Marlinspike, Moxie, (2009) "New tricks for defeating SSL in practice", BlackHat DC.

[12] Shamal, S., Monika, K., \& Neha, N., (2014) "Secure Authentication for Online Banking Using QR Code", IJETAE-International Journal for Emerging Technology and Advance Engineering

[13] Chow, S., Eisen, P., Johnson, H., \& Van Oorschot, P. C., (2003) "White-box cryptography and an AES implementation", Selected Areas in Cryptography, pp250-270.

[14] Winter, J., (2008) "Trusted computing building blocks for embedded linux-based ARM trustzone platforms", Proceedings of the 3rd ACM workshop on Scalable trusted computing, pp21-30.

[15] Chow, S., Gu, Y., Johnson, H., \& Zakharov, V. A., (2001) "An approach to the obfuscation of control-flow of sequential computer programs", Information Security, pp144-155.

[16] Chin, E., Felt, A. P., Greenwood, K., \& Wagner, D., (2011) "Analyzing inter-application communication in Android", Proceedings of the 9th international conference on Mobile systems, applications, and services, pp239-252.

[17] Enck, W., Gilbert, P., Han, S., Tendulkar, V., Chun, B. G., (2014) "TaintDroid: an information-flow tracking system for realtime privacy monitoring on smartphones", ACM Transactions on Computer Systems.

[18] Kruegel, C., Robertson, W., \& Vigna, G., (2004), "Detecting kernel-level rootkits through binary analysis", Computer Security Applications Conference, pp91-100.

[19] Park, J., Park, M., \& Jung, S., (2013). "A whitelist-based scheme for detecting and preventing unauthorized AP access using mobile device", The Journal of Korean Institute of Communications and Information Sciences, No.38, pp632-640. 